\title{
Barreiras e facilitadores no manejo da sede pré-operatória do paciente queimado à luz do Knowledge Translation*
}

\author{
Barriers and facilitators in the management of preoperative thirst of \\ the burned patient in the light of Knowledge Translation \\ Barreras y facilitadores en el manejo de la sede pre operatoria del \\ paciente quemado a la luz del Knowledge Translation
}

Como citar este artigo:

Garcia AKA, Conchon MF, Pierotti I, Zampar EF, Fonseca LF. Barriers and facilitators in the management of preoperative thirst of the burned patient in the light of Knowledge Translation. Rev Esc Enferm USP. 2021;55:e03764. https://doi.org/10.1590/S1980-220X2020039803764

\section{Aline Korki Arrabal Garcia ${ }^{1}$ \\ Marilia Ferrari Conchon ${ }^{1}$ \\ Isadora Pierotti ${ }^{1}$ \\ Elisangela Flauzino Zampar ${ }^{1}$ \\ Lígia Fahl Fonseca ${ }^{1}$}

* Extraído da tese: "Implantação do Modelo de Manejo da Sede Pré-operatória no Centro de Tratamento de Queimados: estudo de intervenção norteado pelo Knowledge Translation", Programa de Pós-Graduação em Enfermagem Universidade Estadual de Londrina, 2020.

${ }^{1}$ Universidade Estadual de Londrina,

Programa de Pós-graduação em

Enfermagem, Londrina, PR, Brasil.

\begin{abstract}
Objective: To explore the perception of health care professionals about barriers and facilitators, and coping strategies for the implementation of the Thirst Management Model in the preoperative period of the burned patient. Method: This is a qualitative study, anchored in the conceptual framework Knowledge Translation. The focus group technique was chosen for data collection, composed by eight key professionals, to identify barriers and facilitators in evidence implementation and to point out coping strategies for the critical nodes found. Thematic Content Analysis technique was used to evaluate the data. Results: Five categories emerged: Physical structure, environment and supplies; Particularities of the burned patient; Human Resources; Team attitude; Team training and education. Coping strategies were raised for each barrier identified. Conclusion: The main barriers identified were found in the Human Resources and Team Attitude categories. The main facilitators were Particularities of the burned patient and Team training and education. The identification allowed planning coping strategies for critical nodes, providing support for scientific evidence in clinical practice.
\end{abstract}

\section{DESCRIPTORS}

Evidence-Based Nursing; Translational Medical Research; Perioperative Nursing; Burns; Thirst. 


\section{INTRODUÇÃO}

Uma enfermagem forte e inovadora se faz por meio da implantação de evidências científicas e mudanças na prática. No momento em que se discute e reflete a respeito da relevância da enfermagem no cenário mundial, a simples divulgação de resultados de pesquisas não garante sua utilização na prática clínica. Esta é uma constatação que redireciona o significado de se fazer pesquisa ${ }^{(1)}$. Nessa vertente, a Organização Mundial de Saúde chama a atenção para a necessidade de traduzir o conhecimento em ação para melhorar a saúde pública, fazendo a ponte entre o que é conhecido e o que é realmente feito ${ }^{(2)}$.

Visando suprir recursos que instrumentalizem o enfrentamento desse problema, desenvolveu-se o referencial teórico Knowledge Translation (KT), que enfatiza modelos de articulação e intercâmbio entre a produção de evidências científicas e as práticas assistenciais ${ }^{(3)}$. O KT preconiza a utilização de seis elementos essenciais: uso de evidência científica, conhecimento do cenário/contexto, uso de intervenções multifacetadas, identificação de barreiras e facilitadores para implantação do uso da evidência, avalição/auditorias e sustentabilidade da implantação(4).

Destaca-se neste artigo a exploração do elemento essencial "identificação de barreiras e facilitadores" em um cenário inédito de implantação do Modelo de Manejo da Sede (MMS) no paciente cirúrgico queimado. A relevância de se explicitar as barreiras e facilitadores, dentro do referencial teórico KT, justifica-se por sua influência na escolha das estratégias de enfrentamento a serem utilizadas para a transferência do conhecimento ${ }^{(5)}$. Para que essa identificação ocorra, deve-se reunir grupo seleto de profissionais a fim de identificarem conjuntamente as principais barreiras e facilitadores e estabelecerem estratégias de enfrentamento para uma implantação bem-sucedida ${ }^{(6)}$.

Considerando que o KT possibilita a realização de mudanças efetivas na prática clínica, ele torna-se útil em cenários complexos e ainda pouco explorados, como na questão da sede do paciente cirúrgico queimado em período pré-anestésico. Embora pouco valorizada na prática clínica, a sede apresenta-se prevalente, intensa, desconfortante e ainda não faz parte dos protocolos de cuidado a este grupo de pacientes ${ }^{(7)}$. Mesmo que existam evidências consistentes e robustas sobre a possibilidade da abreviação do tempo de jejum $^{(8)}$, elas ainda são pouco difundidas na prática cirúrgica.

No paciente cirúrgico queimado, a sede agrava-se. Isso ocorre porque, além dos fatores a que um paciente cirúrgico comum é submetido, o paciente queimado pode ter que realizar múltiplas intervenções para o gerenciamento de suas lesões: balneoterapias para banho e troca de curativos sob sedação, desbridamentos e enxertos, requerendo jejum para cada um desses procedimentos. Além disso, a própria queimadura desencadeia reações fisiológicas, provocando edema e, por consequência, hipovolemia ${ }^{(8)}$. Paradoxalmente, não se localizaram estudos abordando especificamente a sede do paciente queimado na literatura nacional e internacional ${ }^{(8)}$.

Estudos recentes em fisiologia da sensação, acrescidos de evidências tanto para identificação como tratamento da sede, resultaram em um MMS perioperatório, inicialmente utilizado em Sala de Recuperação Anestésica $(\mathrm{SRA})^{(9)}$. Trata-se de um modelo fundamentado em quatro pilares: identificação da sede, mensuração, avaliação da segurança e oferta de estratégia de alívio da sede. Ressalta-se que o modelo já foi implantado em instituições públicas e privadas no pré e pós-operatório imediato (POI), conferindo maior segurança e conforto ao paciente.

A necessidade de intervir na sede do paciente cirúrgico queimado motivou a utilização do MMS neste cenário, considerando sua ampla aplicabilidade clínica. Nesse contexto, o uso do referencial teórico KT é aplicável e necessário para uma implantação efetiva, já que envolve a investigação e intervenção sobre os principais nós críticos, entendidos como barreiras que dificultam a implementação eficaz das evidências científicas ${ }^{(1)}$.

Esta pesquisa, portanto, tem como objetivo explorar a percepção dos profissionais de saúde sobre barreiras e facilitadores e as estratégias de enfrentamento para a implantação do MMS no pré-operatório do paciente queimado.

\section{MÉTODO}

\section{TIPO DE ESTUDO}

Estudo descritivo, com abordagem qualitativa, ancorado no referencial teórico KT, que foi utilizado prospectivamente no desenvolvimento dos grupos focais e análise dos dados ${ }^{(4)}$.

\section{Cenário}

A unidade que recebeu a implantação da evidência foi um Centro de Tratamento de Queimados (CTQ), referência no norte do Paraná, que conta com duas salas cirúrgicas e apresenta média mensal de 69 cirurgias e uma sala de balneoterapia que realiza em média 122 procedimentos mensais. O CTQ conta com 15 leitos de enfermaria e seis leitos de unidade de tratamento intensivo (UTI). Para a realização de todas as atividades desse setor, a equipe é composta por oito enfermeiros e 33 técnicos de enfermagem, distribuídos nos turnos manhã, tarde e noite.

A implantação do MMS que visa a identificar, mensurar, avaliar a segurança e aliviar a sede por meio de uma estratégia ${ }^{(9)}$ ocorreu no período pré-operatório. Este período foi selecionado devido aos múltiplos e sequenciais procedimentos a que o paciente queimado é submetido, fazendo com que ele permaneça em jejum pré-anestésico com frequência e por períodos prolongados.

Anteriormente à fase de implantação, a pesquisadora realizou uma imersão na realidade do setor por um mês. Acompanhou a rotina da unidade, conhecendo o perfil dos trabalhadores e pacientes, bem como os procedimentos realizados, a fim de que os profissionais se familiarizassem com ela.

\section{Critérios de SeleÇão}

Compuseram a amostra oito profissionais de saúde, entre docentes e trabalhadores do CTQ: uma enfermeira assistencial (EA) e uma gestora (EG) da unidade, duas técnicas de enfermagem (TE1 e 2), um cirurgião chefe da unidade (C), 
um anestesiologista (A) e duas docentes em enfermagem e doutoras na temática sede (D1 e 2). A amostra foi intencional e os critérios de inclusão foram determinados em virtude de suas experiências profissionais na temática sede ou assistência com paciente queimado e inserção representativa na unidade de escolha, de forma a atuarem como membros facilitadores na implantação.

A escolha pelo grupo focal para a coleta de dados deu-se pelo fato de proporcionar uma interação grupal para a produção de dados acerca dos objetivos do estudo ${ }^{(10)}$.

\section{Coleta de Dados}

Não foi realizado estudo piloto por se entender que os profissionais que precisariam compor o grupo focal eram ímpares no setor. A coleta de dados foi feita em duas etapas descritas a seguir, que ocorreram após o aceite da participação, em outubro de 2019.

A primeira etapa foi composta por uma sessão com duração de aproximadamente uma hora e meia, realizada em uma sala de reunião confortável e privada dentro do CTQ , com a participação de todos os membros convidados. A moderadora foi a pesquisadora principal, que é especialista e mestra em enfermagem perioperatória e sede. A pesquisadora principal e o auxiliar foram capacitados por meio de intervenção educativa sobre o referencial teórico, MMS e técnicas de coleta e registro de dados para a condução do grupo focal, totalizando oito horas.

Ainda na primeira sessão foi realizada uma apresentação dialogada, expondo o MMS pré-operatório e as evidências que o embasam. Subsequentemente, foram apresentados aos participantes os dados basais auditados no período pré-implantação. Os dados foram auditados pela pesquisadora principal durante dois meses, três dias da semana, nos diversos períodos de trabalho, por meio de observação direta e coleta em prontuário. Estes dados referem-se ao tempo de jejum pré-operatório para sólidos e líquidos, média de intensidade de sede no adulto e criança, sinais periféricos relacionados à sede que os pacientes apresentaram, porcentagem de pacientes que sentiram sede e não verbalizaram espontaneamente e porcentagem de profissionais que perguntaram ao paciente se estava com sede.

Após esse primeiro momento, os participantes foram estimulados a falarem sobre as possíveis barreiras e facilitadores que eles identificavam para a implantação do MMS. Posterior a esse espaço de reflexão e discussão, os convidados escreveram suas respostas em papéis adesivos. A partir delas, foi construído um quadro que reuniu todas as barreiras $\mathrm{e}$ facilitadores para a implantação dos quatro pilares do MMS.

Outro momento que ocorreu na primeira sessão foi uma discussão de forma livre das estratégias de enfrentamento que poderiam ser utilizadas para intervir sobre os nós críticos identificados. As estratégias foram registradas no diário de momento ${ }^{(11)}$ da pesquisadora. Para isso, a pesquisadora contou com a ajuda de um pesquisador auxiliar previamente capacitado.

$\mathrm{Na}$ segunda etapa houve uma sessão de aproximadamente uma hora, na qual o objetivo foi apresentar aos participantes os quadros construídos para a validação das barreiras, facilitadores, nós críticos e estratégias de enfrentamento propostas pelo grupo.

\section{ANÁLISE E TRATAMENTO DOS DADOS}

Optou-se pela não realização de novos grupos focais após a pesquisadora identificar a repetição teórica dos dados, ou seja, não haveria fornecimento de novos elementos para aprofundar a teorização ${ }^{(12)}$. Não foi utilizado software específico para o gerenciamento dos dados, apenas o Microsoft Word para síntese e construção dos resultados.

Para análise dos dados, utilizou-se a técnica de Análise de Conteúdo Temática de Bardin ${ }^{(13)}$. Essa análise acontece em três fases: pré-análise, na qual se procede à escolha dos documentos, formulação de objetivos e preparação do material para análise; exploração do material, momento em que os dados brutos são transformados de forma organizada e agregados em unidades de registro; e tratamento dos resultados, etapa na qual é realizada a inferência e interpretação dos dados ${ }^{(13)}$.

A fase de pré-análise aconteceu logo após a realização de cada sessão, onde a pesquisadora analisou os dados de maneira sistemática. $\mathrm{Na}$ fase de exploração, foram realizados possíveis agrupamentos, por meio da construção de quadros de barreiras, facilitadores e estratégias de enfrentamento dos nós críticos, que haviam sido discutidos na primeira sessão, para que fossem validados junto aos participantes. No tratamento dos resultados, foi realizada a interpretação dos dados obtidos e a validação das cinco categorias que emergiram: Estrutura física, ambiente e insumos; Particularidades do paciente queimado; Recursos humanos; Atitude da equipe; Capacitação e educação da equipe.

\section{Aspectos éticos}

A pesquisa atendeu a todas as normas preconizadas pela Resolução n 466/2012 do Conselho Nacional de Saúde, sendo aprovado pelo Comitê de Ética em Pesquisa em seres humanos, sob o CAAE $\mathrm{n}^{\circ}$ 13638519.1.0000.5231 e parecer $\mathrm{n}^{\circ} 3476724$ do ano de 2019.

Os profissionais foram pessoalmente convidados a participar, e a formalização realizada por convite impresso e via e-mail. Todos assinaram o termo de consentimento livre e esclarecido após aceite.

\section{RESULTADOS}

A idade média dos oito participantes era de 47 anos, sendo desse total seis mulheres. Dois eram docentes em enfermagem perioperatória e os demais profissionais atuantes no CTQ há cerca de oito anos, em média. Em relação à titulação dos profissionais, uma técnica de enfermagem apresentava nível médio; outra, graduação. Uma enfermeira e dois médicos eram pós-graduados, uma enfermeira era mestra, e duas docentes eram doutoras. Dois tinham vínculo terceirizado com a instituição em estudo, e dois participantes tinham mais de um emprego.

Construiu-se um quadro (Quadro 1) com a categorização das principais barreiras e facilitadores identificados para a implantação do MMS, além das estratégias de enfrentamento dos nós críticos identificados durante o desenvolvimento dos grupos focais. 
Quadro 1 - Categorização das barreiras e facilitadores e estratégias de enfrentamento para os nós críticos identificados no grupo focal. Londrina, PR, Brasil, 2019.

\begin{tabular}{|c|c|c|c|}
\hline Categorias & Facilitadores & Barreiras & Estratégias de enfrentamento \\
\hline $\begin{array}{l}\text { Estrutura física, } \\
\text { ambiente e } \\
\text { insumos }\end{array}$ & $\begin{array}{l}\text { Presença de freezer no setor para } \\
\text { acondicionar o picolé (A; C; EA; EG; D1; } \\
\text { D2; TE1; TE2). } \\
\text { Setor isolado permite que funcionários } \\
\text { engajados colaborem com outros } \\
\text { colegas (C; EG; TE 1). } \\
\text { Viabilidade de padronizar insumos } \\
\text { aplicados como estratégias de manejo da } \\
\text { sede (EG; C; D1; D2). }\end{array}$ & $\begin{array}{l}\text { Dificuldade de organização } \\
\text { prévia por falta de horário } \\
\text { definido (sequência) } \\
\text { dos procedimentos } \\
\text { anestésico-cirúrgicos (EA; EG; } \\
\text { TE1; TE2). }\end{array}$ & $\begin{array}{l}\text { Cirurgião chefe da unidade encarregou-se de } \\
\text { definir todos os dias os horários dos três primeiros } \\
\text { pacientes }(C) \text {. }\end{array}$ \\
\hline $\begin{array}{l}\text { Particularidades } \\
\text { do paciente } \\
\text { queimado }\end{array}$ & $\begin{array}{l}\text { Profissionais do setor têm percepção } \\
\text { da intensidade da sede do paciente } \\
\text { queimado, o que gera sensibilização (C; } \\
\text { EG; TE 2). }\end{array}$ & - & - \\
\hline $\begin{array}{l}\text { Recursos } \\
\text { humanos }\end{array}$ & $\begin{array}{l}\text { Disponibilidade de um profissional } \\
\text { técnico de enfermagem para confecção } \\
\text { dos picolés (EG; TE1;TE2). }\end{array}$ & $\begin{array}{l}\text { Falta de tempo para o } \\
\text { profissional aplicar o manejo } \\
\text { da sede por depender do } \\
\text { número de pacientes sob sua } \\
\text { responsabilidade (EA; TE1; TE2). }\end{array}$ & $\begin{array}{l}\text { Ao término da capacitação, os servidores concluíram } \\
\text { que conseguem realizar rapidamente o manejo } \\
\text { da sede, durante o tempo em que estão prestando } \\
\text { cuidados básicos (EA; TE1; TE2). }\end{array}$ \\
\hline $\begin{array}{l}\text { Atitude da } \\
\text { equipe }\end{array}$ & $\begin{array}{l}\text { Implantação de evidência científica } \\
\text { benéfica ao paciente (A; C; EA; EG; D1; } \\
\text { D2; TE1;TE2). }\end{array}$ & $\begin{array}{l}\text { Resistência por parte de alguns } \\
\text { anestesistas da equipe quanto à } \\
\text { possibilidade de abreviação do } \\
\text { jejum }(\mathrm{A} ; \mathrm{C} \text {; EA; EG; D1; D2; } \\
\text { TE1; TE2). } \\
\text { Falta de interesse da equipe } \\
\text { perioperatória em aprender e } \\
\text { realizar o manejo da sede pré- } \\
\text { operatória (EA; EG; TE1; TE2). }\end{array}$ & $\begin{array}{l}\text { Reunião com a direção do hospital e chefia da } \\
\text { anestesiologia para apresentação do projeto, } \\
\text { exposição das evidências sobre o benefício do } \\
\text { manejo da sede, redução do jejum e apoio ao } \\
\text { projeto (D1; D2; A; C; EG). } \\
\text { Divulgação do projeto e seu objetivo por meio } \\
\text { de estratégias personalizadas para o setor, que } \\
\text { contemplasse profissionais e pacientes (D1; D2; EG). } \\
\text { Exposição dos dados da coleta basal para } \\
\text { sensibilização dos profissionais ao tema (D1; D2). }\end{array}$ \\
\hline $\begin{array}{l}\text { Capacitação e } \\
\text { educação da } \\
\text { equipe }\end{array}$ & $\begin{array}{l}\text { Disposição da equipe de implantação } \\
\text { do manejo da sede em capacitar toda a } \\
\text { equipe que presta cuidados ao paciente } \\
\text { (C; EG; D1; D2). }\end{array}$ & $\begin{array}{l}\text { Falta de conhecimento da } \\
\text { equipe sobre manejo da sede } \\
\text { pré-operatória (A; C; EA; EG; } \\
\text { D1; D2; TE1; TE2). } \\
\text { Aplicação do protocolo para } \\
\text { criança realizada apenas por } \\
\text { enfermeiros, por ser mais } \\
\text { complexa (EA; EG; TE1; TE2). }\end{array}$ & $\begin{array}{l}\text { Capacitações na unidade nos períodos da manhã, } \\
\text { tarde e noite, em diferentes horários, de forma a } \\
\text { contemplar a maioria dos servidores (EA; EG; C; A; } \\
\text { TE1; TE2; D1; D2). } \\
\text { Capacitações dos profissionais para o uso da nova } \\
\text { ficha perioperatória, que contempla o registro do } \\
\text { manejo da sede (EA; EG; D1; D2). } \\
\text { Capacitações individuais de todos os técnicos e } \\
\text { enfermeiros sobre o manejo da sede (D1; D2; EG). } \\
\text { Desenvolvimento e divulgação de vídeo didático } \\
\text { sobre o Modelo de Manejo da Sede adulto e } \\
\text { pediátrico (D1; D2). }\end{array}$ \\
\hline
\end{tabular}

$\mathrm{n}=8$

Enfermeira Assistencial (EA); Enfermeira Gestora (EG); Técnica de Enfermagem (TE1 e TE2); Cirurgião (C); Anestesista (A); Docentes (D1 e D2)

\section{DISCUSSÃO}

A importância deste estudo reside na identificação de barreiras e facilitadores em uma instituição pública de saúde para a adoção de evidências científicas na prática clínica, assim como na análise das estratégias de enfrentamento adotadas para a implantação do MMS no pré-operatório do paciente queimado. Reconhecer barreiras e facilitadores como elemento principal durante a etapa de planejamento da transferência do conhecimento pode ser a chave para que efetivamente as recomendações, validadas por resultados de pesquisas, sejam adotadas na prestação de cuidados assistenciais ${ }^{(14-16)}$. Isso é particularmente importante quando se busca transferir conhecimento sobre um tema inexplorado e possivelmente subvalorizado pelos profissionais assistenciais.

A relevância da construção do quadro com barreiras e facilitadores, percebidos pelos participantes do grupo focal para a implantação do MMS no pré-operatório, encontra-se na necessidade de se entender quais as fragilidades e potencialidades do setor. A compreensão desse contexto subsidiou as decisões, na elaboração das metas e estratégias efetivas, para a transferência do conhecimento a serem implementadas na prática clínica dessa unidade.

É imprescindível que se avaliem barreiras e facilitadores do cenário em questão durante um processo de translação do conhecimento, com subsequente elaboração de estratégias personalizadas e embasamento teórico para abordar esses pontos. A adoção dessa abordagem pode influenciar positivamente a prática baseada em evidência ${ }^{(15-16)}$.

A definição de como o conhecimento deve ser transferido envolve o planejamento cuidadoso desse processo $^{(16-17)}$. Para tal, devem ser considerados fatores que garantam a melhor forma de transferência desse conhecimento. Diferentes modelos de KT estão disponíveis na literatura ${ }^{(18)}$, sendo que barreiras e facilitadores são sempre considerados como influenciadores na escolha das intervenções a serem utilizadas na transferência desse conhecimento ${ }^{(5)}$.

$\mathrm{Na}$ categoria Estrutura física, ambiente e insumos, os profissionais elencaram como barreira o fato de a unidade não ter uma sequência de procedimentos e horários definidos para as cirurgias e balneoterapias, o que dificulta estabelecer 
tempo individual de jejum de duas horas para líquidos claros sem resíduos ${ }^{(19)}$.

$\mathrm{Na}$ categoria Particularidades do paciente queimado, o grupo apontou o fato dessa população sentir muita sede, sensibilizando a equipe. Embora não tenha sido identificado na literatura estudo sobre prevalência ou percepção do paciente queimado quanto à sede, é possível observar na prática como este sintoma está presente nesta população ${ }^{(8)}$. A dicotomia manifestada na prática clínica, entre a sede vivenciada pelo paciente queimado e a lacuna de pesquisas científicas sobre o tema na literatura, evidencia a relevância da realização deste estudo e sua contribuição para a área da enfermagem.

Já na categoria Recursos humanos, apontaram a falta de tempo em razão da sobrecarga de trabalho pelo número reduzido de servidores ${ }^{(20-22)}$. Todavia, essa é a barreira mais comumente identificada pelos profissionais da enfermagem para realizar o KT de forma efetiva, sobretudo nas áreas clínicas com intensa carga de trabalho ${ }^{(20-22)}$.

Embora não identificado a princípio pelo grupo como um facilitador, a participação efetiva de elementos da equipe com cargos de gestão potencializa a coordenação e integração dos diferentes mecanismos, que equilibram a aplicação e implementação, para apoiar e facilitar a equipe a praticar o uso da evidência $^{(21)}$. Conclusão semelhante é apresentada em uma revisão do papel da liderança do enfermeiro na promoção e manutenção da prática baseada em evidência (PBE), que identifica o gerente da unidade como o precursor da $\mathrm{PBE}^{(23)}$.

As barreiras relatadas nas categorias Atitude da equipe e Capacitação e educação da equipe vêm ao encontro de outros estudos que utilizaram o KT para a implantação de evidências na prática. Esses tópicos apontam a cultura organizacional, a crença dos profissionais, o pouco acesso à pesquisa, assim como a falta de sua apropriação, como fatores relacionados à dificuldade de aplicação do conhecimento na prática $^{(24-26)}$. Além disso, a falta de preparo educacional dos profissionais da saúde para a implementação das evidências na prática também é considerada uma barreira ${ }^{(21)}$.

A utilização efetiva da pesquisa primária depende do preparo técnico e da habilidade do profissional, de fatores organizacionais de suporte, do envolvimento contínuo dos usuários do conhecimento, das parcerias estabelecidas, do contexto da instituição na qual será implantada a evidência, além, é claro, da existência de facilitadores ${ }^{(2,20,22,27)}$.

Outra importante barreira apontada foi relacionada aos anestesiologistas, em razão da resistência em abreviar o jejum pré-operatório. Ainda nessa vertente, os profissionais relataram que, na realidade do CTQ, era frequente suspender ou adiar o procedimento anestésico-cirúrgico quando o paciente relatava ter mascado goma ou ingerido água no período pré-operatório - estratégias ofertadas ao paciente no período de jejum pré-anestésico durante o manejo da sede ${ }^{(9)}$. Isso acontecia mesmo que o paciente já estivesse com duas horas de jejum absoluto entre a ingestão e o procedimento.

Como estratégias de KT para transpor essa barreira, fez-se uma reunião com a chefia do serviço de anestesiologia da instituição, quando o projeto "Paciente Sem Sede" (PASS) foi formalmente apresentado. Nesse encontro foram expostas evidências disponíveis na literatura sobre os benefícios da redução do tempo de jejum, além das estratégias possíveis de serem utilizadas no manejo da sede. Essa reunião foi esclarecedora para os anestesiologistas quanto ao objetivo e benefícios da implantação do MMS no setor, transformando esses profissionais de barreira em facilitadores e apoiadores da continuidade do projeto.

Com a intenção de obter o apoio formal da instituição, fez-se outra reunião para apresentação do projeto PASS, com convite à direção do hospital. Para divulgar o projeto, também houve entrevistas para a rádio e jornal da universidade e do hospital, além da distribuição no hospital de banners, cartazes, adesivos e ímãs com a logomarca do projeto.

As intervenções multifacetadas de KT, escolhidas para transpor as barreiras, levaram em consideração evidências que demonstram que intervenções são mais efetivas quando incluem encontros educacionais, associados com reuniões interativas e didáticas, além de auditoria e feedback ${ }^{(5,28)}$.

Diante das barreiras e dos facilitadores identificados nas categorias, foram estabelecidas em conjunto com os profissionais convidados, estratégias de KT multifacetadas dentro de um cronograma de intervenção. Para tal, foram realizadas 55 capacitações dentro do CTQ nos períodos da manhã, tarde e noite, em diferentes horários, de forma a contemplar os servidores (33 técnicos de enfermagem e oito enfermeiros). As capacitações abordaram essencialmente as principais evidências que permitem a abreviação do tempo de jejum, o passo a passo para a aplicação do MMS pré-operatório, além dos dados basais do setor relacionados à sede, para a sensibilização da equipe. Todos os profissionais também foram capacitados para a utilização da nova ficha perioperatória, que foi desenvolvida pela pesquisadora para contemplar o registro do MMS.

Em relação às barreiras encontradas nas categorias Recursos humanos, Atitude da equipe e Capacitação e educação da equipe, uma das dificuldades enfrentadas ao longo das capacitações foi o fato de os funcionários não comparecerem no setor para capacitações fora do horário de serviço. Dessa forma, foi necessário realizá-las durante seus respectivos horários de trabalho. Em alguns momentos, isso fez com que os profissionais tivessem a atenção diminuída pela preocupação com o serviço, pois a unidade já atua com um dimensionamento reduzido de técnicos de enfermagem e enfermeiros. Contextos como esse, que são direcionados para a realização de tarefas, com poucos recursos e estruturas ineficientes, são menos receptivos à mudança ${ }^{(29)}$.

Outro ponto que pode ter dificultado a capacitação foi que as equipes da noite não priorizaram um tempo do plantão para receberem as capacitações. Por esse motivo, quando a pesquisadora se apresentava para o enfermeiro, naquele período na unidade, o profissional solicitava agilidade e uso do ambiente da própria unidade do CTQ para a realização da capacitação. Consequentemente, os servidores desprendiam a atenção a todo momento, pois trata-se de uma unidade com pacientes críticos, que necessitam de cuidados intensivos. Contudo, é importante destacar que, mesmo com essas dificuldades, foi possível realizar capacitações com esses funcionários, com estratégias variadas e em momentos alternados, já considerando uma das premissas do KT, que é conhecer o cenário onde se deseja realizar uma implantação ${ }^{(30)}$. 
Aos servidores que tiveram sua atenção reduzida nas capacitações, o conhecimento inicial foi transmitido juntamente com as ações seguintes do projeto de implantação, por meio de diversas atividades de KT. Isso incluiu a presença do grupo de pesquisa, com estratégias que variaram desde a participação dos "doutores da alegria" local a cantatas com funcionários e pacientes. Além disso, a pesquisadora, com o auxilio de membros do grupo de pesquisa, esteve presente em diversos momentos e em horários alternados dentro do setor, para realizar a capacitação individual do profissional, estimular a aplicação do MMS no período pré-operatório e sanar dúvidas. Também foi possível desenvolver um vídeo explicativo do modelo, tanto para o paciente adulto quanto para o pediátrico.

O referencial teórico KT mostrou-se um arcabouço relevante para a implantação pioneira do MMS na prática clínica do paciente queimado. Esse processo possibilitou a análise e transposição das barreiras, assim como a potencialização de facilitadores. Além disso, essa interface possibilitou maior autonomia e empoderamento do enfermeiro na condução do cuidado humanizado ao paciente queimado com sede.
Como limitador desse estudo, aponta-se a multidisciplinaridade da composição do grupo focal, que pode ter influenciado na liberdade de expressão de alguns participantes.

\section{CONCLUSÃO}

Ao utilizar o referencial teórico KT, foi possível explorar, com a realização dos grupos focais, as principais barreiras, os facilitadores e suas estratégias de enfrentamento para a implantação pré-operatória efetiva do MMS no paciente queimado. As principais barreiras identificadas foram em relação aos tópicos Recursos humanos e Atitude da equipe. Já os principais facilitadores concentraram-se nos itens Particularidades do paciente queimado e Capacitação e educação da equipe. Essa identificação foi essencial para sustentar a resolução de todos os nós críticos, por meio de estratégias personalizadas e efetivas, possibilitando o adequado planejamento da implantação da evidência na prática clínica, de forma que puderam ser realmente adotadas como protocolos assistenciais na unidade estudada.

\section{RESUMO}

Objetivo: Explorar a percepção dos profissionais de saúde sobre barreiras e facilitadores e as estratégias de enfrentamento para a implantação do Modelo de Manejo da Sede no pré-operatório do paciente queimado. Método: Qualitativo, ancorado no referencial teórico Knowledge Translation. A técnica de grupo focal foi escolhida para coleta de dados, sendo formada por oito profissionais-chave, a fim de identificarem barreiras e facilitadores na implantação da evidência e apontarem estratégias de enfrentamento para os nós críticos encontrados. Utilizou-se a técnica de Análise de Conteúdo Temática para avaliar os dados. Resultados: Emergiram cinco categorias: Estrutura física, ambiente e insumos; Particularidades do paciente queimado; Recursos humanos; Atitude da equipe; Capacitação e educação da equipe. As estratégias de enfrentamento foram levantadas para cada barreira identificada. Conclusão: As principais barreiras identificadas foram encontradas nas categorias Recursos humanos e Atitude da equipe. Já os principais facilitadores foram Particularidades do paciente queimado e Capacitação e educação da equipe. A identificação possibilitou o planejamento das estratégias de enfrentamento sobre nós críticos, proporcionando a sustentação das evidências científicas na prática clínica.

\section{DESCRITORES}

Enfermagem Baseada em Evidências; Pesquisa Médica Translacional; Enfermagem Perioperatória; Queimaduras; Sede.

\section{RESUMEN}

Objetivo: Explotar la percepción de los profesionales de salud sobre barreras y facilitadores y las estrategias de enfrentamiento para la implantación del Modelo de Manejo de la Sede en la fase pre operatoria del paciente quemado. Método: Cualitativo, basado en el modelo teórico Knowledge Translation. Se eligió la técnica de grupo focal para recopilación de datos, compuesta de ocho profesionales, con la finalidad de identificar barreras y facilitadores en la implantación de la evidencia e identificar estrategias de enfrentamiento para los ejes críticos encontrados. Se utilizó la técnica de Análisis de Contenido Temático para evaluar los datos. Resultados: se produjeron cinco clases: Estructura física, ambiente y materiales: Particularidades del paciente quemado; Recursos humanos, Actitud del equipo; Capacitación y Educación del equipo. Se crearon estrategias de enfrentamiento para cada barrera identificada. Conclusión: las principales barreras fueron identificadas en las categorías Recursos Humanos y Actitud del equipo. Sin embargo, los principales facilitadores fueron Particularidades del paciente quemado y Capacitación y Educación del equipo. Identificarlo posibilitó la planificación de las estrategias de enfrentamiento sobre los ejes críticos además de sostener las evidencias científicas en la práctica clínica.

\section{DESCRIPTORES}

Enfermería Basada en la Evidencia; Investigación en Medicina Traslacional; Enfermería Perioperatoria; Quemaduras; Sed.

\section{REFERÊNCIAS}

1. Sturke R, Harmston C, Simonds RJ, Mofenson LM, Siberry GK, Watts DH, et al. A multi-disciplinary approach to implementation science: the NIH-PEPFAR PMTCT implementation science alliance. J Acquir Immune Defic Syndr. 2014;67 Suppl 2:S163-7. https://doi.org/10.1097/QAI.0000000000000323

2. World Health Organization. Bridging the "Know-Do" gap meeting on knowledge translation in global health. Geneva: World Health Organization, 2005 [cited 2019 Dec 20]. Available from:https:/www.who.int/bulletin/volumes/83/10/editorial21005html/en/

3. Landry R, Amara N, Pablos-Mendes A, Shademani R, Gold I. The knowledge-value chain: a conceptual framework for knowledge translation in health. Bull World Health Organ. 2006;84(8):597-602. https://doi.org/10.2471/BLT.06.031724.

4. Canadian Institutes of Health Research. More about knowledge translation [Internet]. Ottawa: Canadian Institutes of Health Research; 2017 [cited 2019 Dec 20]. Available from: https://cihr-irsc.gc.ca/e/29529.html

5. Grimshaw JM, Eccles MP, Lavis JN, Hill SJ, Squires JE. Knowledge translation of research findings. Implement Sci. 2012;7(1):50. https://doi.org/10.1186/1748-5908-7-50 
6. Lee SK, Aziz K, Singhal N, Cronin CM, James A, Lee DS, et al. Improving the quality of care for infants: a cluster randomized controlled trial. CMAJ. 2009;181(8):469-76. https://doi.org/10.1503/cmaj.081727

7. Nascimento LA, Nakaya TG, Conchon MF, Garcia AK, Pierotti I, Serato VM, et al. Prevalence, intensity and discomfort of thirst in surgical patients in the immediate post-operative period. Rev SOBECC. 2019;24(2):85-90. https://doi.org/10.5327/Z1414-4425201900020006

8. Togo HY, Lopes EC. Preoperative fasting reduction in burned patients: a systematic review. Burns Open. 2020;4(4):176-82. https://doi.org/10.1016/j.burnso.2020.06.004

9. Nascimento LA, Garcia AK, Conchon MF, Aroni P, Pierotti I, Martins PR, et al. Advances in the management of perioperative patients' thirst. AORN J. 2020;111(2):165-79. https://doi.org/10.1002/aorn.12931

10. Nogueira-Martins MC, Bogus CM. [Considerations on qualitative research as a mean to study health humanization actions]. Saude Soc. 2004;13(3):44-57. Portuguese. https://doi.org/10.1590/S0104-12902004000300006

11. Pezzato LM, L'abbate S. O uso de diários como ferramenta de intervenção da Análise Institucional: Potencializando reflexões no cotidiano da Saúde Bucal Coletiva. Physis. 2011;21(4):1297-314. https://doi.org/10.1590/S0103-73312011000400008

12. Fontanella B], Luchesi BM, Saidel MG, Ricas J, Turato ER, Melo DG. [Sampling in qualitative research: a proposal for procedures to detect theoretical saturation]. Cad Saude Publica. 2011;27(2):388-94. Portuguese. https://doi.org/10.1590/S0102-311X2011000200020

13. Bardin L. Análise de conteúdo. 6th ed. Lisboa: Edições 70; 2016.

14. Harrison MB, Légaré F, Graham ID, Fervers B. Adapting clinical practice guidelines to local context and assessing barriers to their use. CMAJ. 2010;182(2):E78-84. https://doi.org/10.1503/cmaj.081232

15. Légaré F, Zhang P. Barriers and facilitators: strategies for identification and measurement. In: Straus SE, Tetroe J, Graham ID. Knowledge translation in health care: moving from evidence to practice. 2nd ed. London: BMJ Books; 2013. p. 121-36.

16. Dyson J, Lawton R, Jackson C, Cheater F. Development of a theory-based instrument to identify barriers and levers to best hand hygiene practice among healthcare practitioners. Implement Sci. 2013;8(111):111. https://doi.org/10.1186/1748-5908-8-111

17. Lavis JN, Robertson D, Woodside JM, McLeod CB, Abelson J. How can research organizations more effectively transfer research knowledge to decision makers? Milbank Q. 2003;81(2):221-48. https://doi.org/10.1111/1468-0009.t01-1-00052

18. Sudsawad P. Knowledge translation: introduction to models, strategies, and measures [Internet]. Austin: National Center for the Dissemination of Disability Research; 2007 [cited 2019 Dec 26]. Available from: https://ktdrr.org/ktlibrary/articles_pubs/ktmodels/

19. de-Aguilar-Nascimento JE, Salomão AB, Waitzberg DL, Dock-Nascimento DB, Correa MI, Campos AC, et al. ACERTO guidelines of perioperative nutritional interventions in elective general surgery. Rev Col Bras Cir. 2017;44(6):633-48. https://doi.org/10.1590/0100-69912017006003

20. Bezerra LC, Felisberto E, Costa JM, Hartz Z. [Knowledge translation in qualification of health surveillance management: contribution of postgraduate evaluative studies]. Physis. 2019;29(1):e290112. Portuguese. https://doi.org/10.1590/s0103-73312019290112

21. Harvey G, Kelly J, Kitson A, Thornton K, Owen V. Leadership for evidence-based practice - Enforcing or enabling implementation? Collegian. 2020;27(1):57-62. https://doi.org/10.1016/j.colegn.2019.04.004

22. Gagliardi AR, Berta W, Kothari A, Boyko J, Urquhart R. Integrated knowledge translation (IKT) in health care: a scoping review. Implement Sci. 2016;11(38):38. https://doi.org/10.1186/s13012-016-0399-1

23. Bianchi M, Bagnasco A, Bressan V, Barisone M, Timmins F, Rossi S, et al. A review of the role of nurse leadership in promoting and sustaining evidence-based practice. J Nurs Manag. 2018;26(8):918-32. https://doi.org/10.1111/jonm.12638

24. Stevens BJ, Yamada J, Promislow S, Stinson J, Harrison D, Victor JC. Implementation of multidimensional knowledge translation strategies to improve procedural pain in hospitalized children. Implement Sci. 2014;9(120):120. https://doi.org/10.1186/s13012-014-0120-1

25. Puchalski Ritchie LM, Khan S, Moore JE, Timmings C, Lettow M, Vogel JP, et al. Low- and middle-income countries face many common barriers to implementation of maternal health evidence products. J Clin Epidemiol. 2016;76:229-37. https://doi.org/10.1016/j.jclinepi.2016.02.017

26. Abdel Razeq NM. Barriers that impede the provision of pain care to neonates by nurses in Jordan. J Obstet Gynecol Neonatal Nurs. 2016;45(3):371-7. https://doi.org/10.1016/j.jogn.2016.01.007

27. Tricco AC, Cardoso R, Thomas SM, Motiwala S, Sullivan S, Kealey MR, et al. Barriers and facilitators to uptake of systematic reviews by policy makers and health care managers: a scoping review. Implement Sci. 2016;11(4):4. https://doi.org/10.1186/s13012-016-0370-1

28. Saunders H, Gallagher-Ford L, Kvist T, Vehviläinen-Julkunen K. Practicing healthcare professionals' evidence-based practice competencies: an overview of systematic reviews. Worldviews Evid Based Nurs. 2019;16(3):176-85. https://doi.org/10.1111/wvn.12363

29. Stevens B, Riahi S, Cardoso R, Ballantyne M, Yamada J, Beyene J, et al. The influence of context on pain practices in the NICU: perceptions of health care professionals. Qual Health Res. 2011;21(6):757-70. https://doi.org/10.1177/1049732311400628

30. Oelke ND, Lima MAS, Acosta AM. [Knowledge translation: translating research into policy and practice]. Rev Gaúcha Enferm. 2015;36(3):113-7. Portuguese. https://doi.org/10.1590/1983-1447.2015.03.55036

\section{Apoio financeiro}

Coordenadoria de Aperfeiçoamento de Pessoal de Nível Superior (Capes). Proc. 88882448691/2019-01 e 888887465257/2019-00. Conselho Nacional de Desenvolvimento Científico e Tecnológico (CNPQ - processo 425262/2018-9) - Projeto Universal 